\title{
FETO-MATERNAL PROTECTIVE FUNCTION OF PROGESTERONE DURING PARTURITION - A NEW PERSPECTIVE.
}

\author{
Dr. Asha Anand \\ Dharwadkar* \\ Dr. Anand \\ Rajendra \\ Dharwadkar
}

Retired Professor and Head, Department of Human Physiology. *Corresponding Author

Retired Professor and Head, Department of Human Physiology.

ABSTRACT Normal parturition concludes with safe delivery of viable conceptus by forceful emptying of uterus in humans. With already well accepted experimental and clinical observations, new perspective tries to explain it by calcium ions [Ca ${ }^{2+}$ ] lowering action of Progesterone [PR] and Negative feedback regulation of Oestrogen[OE] on Human chorionic gonadotropin[HCG] as follows.

Respiratory centre stimulatory effect of PR causes respiratory alkalosis and consequentially converts biologically active plasma free $\mathrm{Ca}^{2+}$ to its' inactive protein bound store. Thus PR induced low plasma $\mathrm{Ca}^{2}$ maintains pregnancy by supressing myometrial and neural excitability. At term normal fetal organogenesis coordinated with feto-placental steroidogenesis, cause OE negative feedback on HCG, inhibiting both OE and PR synthesis. Due to shorter biological half-life [t/2 = 30min], PR dip is first observed. So the sudden PR withdrawal at term causing acute respiratory acidosis with spark release of plasma $\mathrm{Ca}^{2+}$, facilitates neuro- myometrial excitability triggering parturition. During each myometrial contraction, sandwiched PR gets squeezed from placental store into maternal circulation again resulting in myometrial relaxation. Thus myometrial alternate cyclical repetition of contraction and relaxation [labour pains] continues till completion of parturition.

Awareness of importance of gradual intermittent release of placental PR and spark plasma $\mathrm{Ca}^{2+}$ helps clinicians in prevention of feto-maternal mortality and morbidity including puerperal psychosis associated with clinical conditions of sudden PR withdrawal as in caesarean section, precipitous labour, premature labour, preeclamsia etc. which can be treated by building better body blood buffers.

KEYWORDS : parturition, progesterone block, Oestrogen, Human chorionic gonadotropin, feto-maternal mortality, respiratory alkalosis, calcium trigger

INTRODUCTION -

The following is the summary of the development of understanding of the mechanism of initiation and process of parturition as explained in Volume 2 Sam Mesiano, .. Louis J. Muglia, in Knobil and Neill's Physiology of Reproduction (Fourth Edition), 2015.with cross references

'."The process of parturition in humans is similar to viviparous species and concludes with safe delivery of viable conceptus by the forceful emptying of the uterus . Historically, the central role of the hormone progesterone in maintainance of pregnancy and blockage of the onset of labor, has been recognized for more than 70 years. This "progesterone block" hypothesis, originally proposed by George Corner in the $1940 \mathrm{~s}^{1}$ and later refined by Arpad Csapo in the 1950s, ${ }^{2}$ still is a foundation of parturition physiology. Subsequent in-vivo studies in sheep by Liggins and colleagues in the 1960s, established activation of the fetal hypothalamic-pituitary -adrenal axis as the driver of withdrawing progesterone (removing the block) and initiating parturition. ${ }^{3-5}$ Systemic progesterone withdrawal i.e. a fall in circulating serum progesterone, is not demonstrable in women before the onset of labor ${ }^{6,7}$.

The gravid uterus is controlled by hormones generally classified as uterotropins and uterotonins. Uterotropins (e.g., estrogens and progesterone) establish the phenotype of the uterus by affecting its overall growth, contractile capacity, biomechanical integrity, and inflammatory status. Uterotonins (e.g., prostaglandins (PGs), which are 20-carbon unsaturated fatty acids that exert a spectrum of signaling effects on inflammation, smooth muscle function, and cellular metabolism $^{8-11}$; and oxytocin (OT)) are hormones that directly stimulate or repress myometrial contraction and play critical roles at parturition by inducing the contractions of labor and cervical dilation. For most of pregnancy, pro-gestation uterotropins stimulate the growth of the uterus needed to accommodate the conceptus, and promote myometrial quiescence, closure of the cervix, and inflammatory quiescence at the maternal-fetal interface between the decidua and the fetal membranes. For parturition to occur, the effects of pro-gestation uterotropins (especially progesterone) diminish and the uterus is transformed to the laboring state through the combined effects of pro-labor uterotropins (mainly estrogens and PGs) and stimulatory uterotonins, especially prostaglandin $\mathrm{F} 2$ alpha $\left(\mathrm{PGF}_{2 \mathrm{a}}\right)$, prostaglandin $\mathrm{E} 2$ $\left(\mathrm{PGE}_{2}\right)$, and OT. During this process, the myometrium becomes highly contractile and excitable to produce the coordinated and forceful contractions of labor in response to $\mathrm{PGF}_{2 a}$ and OT. At the same time, the remodeling of the extracellular matrix (ECM) ECM of the cervix remodels in response to $\mathrm{PGE}_{2}$, causing the tissue to become thin, soft, and distensible such that it dilates in response to increased intrauterine pressure induced by each contraction. Eventually, the cervix dilates wide enough to allow passage of the conceptus into the birth canal. Biochemical changes in extracellular matrix (ECM) ECM also occur at the fetal-maternal interface, due to inflammation in the decidua, to cause weakening and rupture of the adjacent fetal membranes (the amnion and chorion). Inflammation in the myometrium, cervix, and decidua is also a major component of the parturition processes and precedes the onset of active labor. There is complex interplay between specific uterotropins, uterotonins, and proinflammatory hormones in the myometrium, cervix, and decidua that induce and mediate parturition". ${ }^{[8-11]}$

The success of pregnancy is dependent on the appropriate timing of parturition. Ideally, birth should occur at a stage in gestation when the fetus is physiologically prepared for life outside of the uterus. Full term for human pregnancy is at the 40th completed week of gestation (calculated from the first day of the mother's last menstrual period). At this time, the probability of the fetus and mother surviving the parturition process is at its apex, with the risk for neonatal and/or maternal morbidity and mortality increasing exponentially the farther birth occurs from term (whether preterm or postterm $)^{[8-11]}$ 
In absence of demonstrable direct action of PR on isolated myometrium the mechanism still remains unclear. New perspective explains "PR block" during pregnancy as PR [t/2 $=30 \mathrm{~min}$ ] induced plasma $\mathrm{Ca}^{2+}$ lowering mechanism and initiation of parturition as PR withdrawal at term.

\section{METHODOLOGY}

This review was prepared based on the papers published in PubMed, Google Scholar, indexed journals and standard anatomy, embryology and physiology textbooks.

\section{The New Perspective -}

It Concentrates On Progesterone [PR] Induced Fluctuations Of

Acid -base Balance Of Body And It's Relation To Plasma Calcium.

Plasma Calcium present in two forms i.e.

- a] 'Ionic form [biological active form]' and

- b] 'Bound form bound to organic and inorganic anions [store form]' are interconvertible, depending on $\mathrm{pH}$ of the environment. i.e.

- the $\mathrm{H}^{+}$of acidic environment competes with calcium in bound form releasing $\mathrm{Ca}^{2+}$ and

- viceversa in alkaline environment which favours conversion of $\mathrm{Ca}^{2+}$ to bound form.

- PR stimulates respiratory center causing acute respiratory alkalosis favouring conversion of active $\mathrm{Ca}^{2+}$ to inactive bound form. and PR withdrawal inhibiting respiratory center cause acute respiratory acidosis ${ }^{[12-16]}$

In Conclusion New Perspective Explains Âs Follows -

- "At full term, due to negative feedback effect of OE on HCG there is diminished synthesis of $\mathrm{OE}$ and PR. As the half-life of circulatory PR is 3-30 min compared to 6-8 hrs of OE, the dip in PR level is first observed. The sudden PR withdrawal inducing acute respiratory acidosis increase biologically active plasma calcium ions. It opposes 'progesterone block' of pregnancy initiating parturition with facilitation of myometrial contractility".

Discussion And Evaluation Of New Perspective-.

Source Of Progesterone -

Progesterone [PR] is a steroid hormone with a CPP [ Cyclo pentano- perhydrophenanthrine] ring synthesised from cholesterol in adrenal cortex, corpus luteum of ovary, placenta. It is the first intermediary product in the biosynthesis of mineralo corticoids, gluco-corticoids, sex steroids like estrogen[OE] and androgens. PR production in turn is regulated by $\mathrm{ACTH}, \mathrm{GnRH}$ of anterior pituitary and HCG of syntitioblast cells i.e. foetal portion of placenta. The half-life of natural PR is very short extending from $5-30$ minutes. PR is known to be the main hormone for maintenance of pregnancy through "progesterone block" which causes depression of myometrial excitability and non-responsiveness of myometrium to natural uterotonics like Oxytocin [OX].

The experimental observation of removal of corpus luteum in termination of early pregnancy has proved the fact. Based on this principle, clinically antiprogestins are used in termination of early pregnancy. After 12 weeks of gestation PR and OE are mainly synthesised in the feto-placental unit. During pregnancy the hypertrophied foetal adrenal cortex $[80 \%$ of which involutes in newborn after birth] and active male foetal testis helps in converting PR to DHEA [Androgen] which gets aromatized in placenta to $\mathrm{OE}$.

With the active participation of foetal organs in steroid hormones synthesis, the quantity of both OE and PR secretions significantly increase with foetal organogenesis from $20^{\text {th }}$ to $40^{\text {th }}$ week of gestation. Near term, at about 24 to 48 hours before onset of parturition, OE attains a peak with simultaneous dip in PR
A] The Experimental Predictive Proofs Of PR Withdrawal -

- 1] fall in BBT $\mathrm{l}^{\circ} \mathrm{C}$ in calfs and bitch $24 \mathrm{hrs}$ before noticed by Concanon et al in 1989 [17]

- 2] in mares - low OE and PR observed near term but 5a PR metabolites production increased near term as observed by Holtan et al in 1975 and $1991 \quad{ }^{[18,19]}$

- 3] Ousey et al in 1884 observed high $\mathrm{Ca}$ and $\mathrm{Na}$ in prepartum milk before parturition which was used as indicator of parturition in a commercially used predictor kit. ${ }^{[20]}$

- Conclusive Highlights Of Already Accepted Observati ons

- Inspite of many studies, the perspective of OE negative feedback regulation on HCG and it's relation to initiation of parturition remains ignored.

- On full response to HCG the OE is end product and PR and DHEA are intermediary products.

- At $40^{\text {th }}$ week of gestation peak of OE and dip of PR indicate fetal viability

- B] Conclusive Highlights of new perspective i.e. PR related calcium lowering mechanism ${ }^{[12-16]}$

- PR stimulates respiratory center causing acute respiratory alkalosis and PR withdrawal results in acute respiratory acidosis.

- Acidosis favours release of active $\mathrm{Ca}^{2+}$ and alkalosis favours lowering of $\mathrm{Ca}^{2+}$

- PR induced respiratory alkalosis causes progesterone block' of pregnancy by reduced active $\mathrm{Ca}^{2+}$

- "Negative feedback effect of OE on HCG at term with resultant reduced circulatory $\mathrm{PR}$, reverses respiratory alkalosis inturn increases plasma calcium level thus opposing 'progesterone block' of pregnancy initiates parturition by fascilitating myometrial contractility".

New Perspective - Tries To Explain The Following Physiological Observations Related To Pregnancy And Parturition.

1] Mechanism of "PR block' during pregnancy.

2] PR fluctuations during pregnancy and at term.

- [both in normal and preeclamsia]

3] Inter-relationship of HCG, OE and PR. Highlighting role of normal viable fetal organogenesis.

- [both in normal and preeclamsia]

4] PR fluctuations at term reverse 'progesterone block' of pregnancy and initiate parturition with plasma calcium level oscillations facilitating myometrial contractility.

- [both in normal and preeclamsia]

5] Mechanism of PR withdrawal induced facilitation of myometrial and cervical excitability with establishment of neuro-endocrinal reflex mediated by Oxytocin .

- Animal expt and clinical evidences of initiation of labour by Oxytocin.

6] Highlights of physiological importance of normal partutition in minimizing maternal and fetal mortality and morbidity.

- Ist stage of labour - aim - gradually reduce the maternal circulating PR level to decrease the maternal morbidity [prevention of thrombo-embolism, puerperal psychosis etc] and mortality due to cardiac arrest as a result of calcium rigor induced by sudden spurt of plasma calcium ions on PR withdrawal.

- $2^{\text {nd }}$ stage of labour - ripening of cervix by facilitating its excitability and ability to respond to Oxytocin.

- Increased fetal general excitability at the time of birth Increased plasma calcium ions transferred from mother to fetus enhances fetal general excitability increasing its response to hypoxia.

- 7] Possible predictions of parturition at term.

8] Further possible studies to strengthen the new perspective

Discussion And Evaluation Of New Perspective-

1] Mechanism Of "PR [Progesterone] block During Pregnancy. 
New perspective tries to explain the "PR block i.e. suppression of myometrial excitability" due to PR induced reduction of active plasma $\mathrm{Ca}^{2+}$ during pregnancy. The details are as follows.

- PlasmaCa ${ }^{2+}$ lowering mechanism of PR is due to PR stimulation of respiratory center causing acute respiratory alkalosis $\rightarrow \downarrow$ in biologically active plasma $\mathrm{Ca}^{2+}$ and $\uparrow$ in plasma protein bound Calcium i.e. stored form of Calcium [Note - the total plasma calcium is maintained in normal physiological range].

- $\quad \downarrow$ Biologically active plasma $\mathrm{Ca}^{2+} \rightarrow$ depression of smooth muscle excitability and contractility $\rightarrow$ generalized smooth muscle relaxation $\rightarrow$ generalized blood vascular dilatation and myometrial relaxation.

- The magnitude of plasma $\mathrm{Ca}^{2+}$ lowering mechanism of PR is directly proportional to PR quantity. As PR level gradually increases from 2 to 40 weeks of gestation there is natural steady build-up of PR block.

\section{CONCLUSION -}

- PR block cause myometrial relaxation accommodating the growing foetus without increase in the intra uterine pressure and thus sustains pregnancy.

- The associated placental vascular dilatation maintains adequate foetal blood supply.

- 2] Mechanism of Acute and chronic respiratory alkalosis status during pregnancy

- It can be explained by the body $\mathrm{pH}$ regulatory mechanism with both respiratory and renal handling as follows -

- PR stimulation of respiratory center $[R C] \rightarrow \uparrow$ respiratory rate $\rightarrow \uparrow \mathrm{CO} 2$ washout $\rightarrow$ acute respiratory alkalosis with $\downarrow \mathrm{pCO} 2 \rightarrow$ chronic respiratory alkalosis by renal handling with an effort to retain $\mathrm{H}+$ ions in the body by reducing tubular $\mathrm{H}+$ ions secretion with less reabsorption of $\mathrm{HCO} 3-$.

- There is gradual steady increase in PR synthesis throughout the 40 weeks of gestation reaching 13 times more comparing to the levels at 4 weeks. The initial respiratory centre stimulation of $\mathrm{PR}$ inducing acute respiratory alkalosis is compensated by renal handling trying to retain $\mathrm{H}+$ ions inturn excreting more $\mathrm{HCO} 3$ - ions with comparative alkaline urine.

- New Perspective Conclusion-So in gestation there is both acute and chronic respiratory alkalosis status and body $\mathrm{pH}$ tries to be regulated with low $\mathrm{pCO} 2$ and low $\mathrm{HCO} 3-$ ions $\rightarrow$

- Resultant reduction in both buffers like HCO3-ions and free plasma proteins [as alkalinity favours calcium binding to proteins].

- Thus the pregnant status makes the body more sensitive to acid-base variations with resultant $\mathrm{Ca} 2+$ spark oscillations and

- During pregnancy the main cause of $\mathrm{pH}$ variation is $\mathrm{PR}$ $[t / 2=30 \mathrm{~min}]$ fluctuations.

3] Inter-relationship of HCG, OE and PR and

- Highlighting the role of normal viable fetal organogen esis.

- Both in normal and preeclamsia.

Accepted observations - Under the influence of HCG from the implantation of conceptus to full term [ 4 to 40 weeks of gestation]

- From 4 to 20 weeks of gestation - Gradual increase in OE and PR synthesis mainly from corpus luteum of ovary and partly by feto-placental unit with predominance of $\mathrm{PR}$ $[\mathrm{PR} / \mathrm{OE}$ ratio $=20]$

- from 20 To 40 Weeks Of Gestation - Steady increase in $\mathrm{OE}$ and $\mathrm{PR}$ synthesis continues by feto-placental unit with predominance of $\mathrm{OE}[\mathrm{PR} / \mathrm{OE}$ ratio $=12]$

- 24- $48 \mathrm{hrs}$ before onset of normal parturition - OE synthesis is at peak with sudden dip in PR. ${ }^{[17-20]}$

Accepted Conclusions -

- Highlights the positive feedback inter-relation of normal viable fetal organogenesis with steroidogenesis of fetoplacental unit.

- The fetal organs like Hypertrophied adrenal cortex and fetal testis take active part in conversion of PR to androgens $\{D H E A\}$ which gets aromatized to $\mathrm{OE}$ in placenta.

- So level of maternal urinary OE metabolic products is said to be predictor of normal fetal organogenesis. ${ }^{[21-23]}$.

- In many studies of pre-eclampsia there is significant association of IUGR [In Utero fetal Growth Retardation] with diminished OE and PR synthesis with heightened HCG levels ${ }^{[24-26]}$

New Perspective Conclusion-

- "There is negative feedback regulation of OE on HCG"

- Revision of Supporting observational proofs -

- Fluctuations of HCG with OE and PR along with PR/OE $\underset{[24-25]}{\text { ratio at different weeks of normal gestation [ref. guyton] }}$

- Figure no. 1 - Modified figure of Fluctuations of HCG with $\mathrm{OE}$ and $\mathrm{PR}$ along with $\mathrm{PR} / \mathrm{OE}$ ratio at different weeks of normal gestation. For easier understanding the levels are expressed as multiples of corresponding values at 4 Weeks. ${ }^{[23]}$

${ }^{i}$ i] Highlights of observations supporting inverse relation of HCG and OE [Fig no 1]:

- From 4 to 10 weeks of gestation - There is sharp rise of HCG to 120 times and OE to 2 times as compared to the values at 4 weeks.

- From 10 to 20 weeks of gestation - HCG falls steadily to 25 times and OE increase to 4 times.

- From 20 to 40 weeks of gestation - Ther is steady state of $\mathrm{HCG}$ at 25 times there is gradual increase of OE synthesis to 20 times

- Strengthening the fact of "Fetal organogenesis participating in feto-placental steroidogenesis".

ii] Highlights Of Observations $\mathrm{Of} P R$ and $P R / O E$ Ratio from 20 to 40 weeks [supporting PR and OE dominant synthesis [dominant PR synthesis shows more ratio and dominant $\mathrm{OE}$ synthesis shows less ratio] [Fig no 1]:

- From 4 to 20 weeks of gestation - PR synthesis gradually increases to 4 times maintaining steady PR/OE ratio of 20 .

- From 20 to 40 weeks of gestation-

- PR synthesis increases to 13 times at 40 weeks with

- gradual decline in PR/OE ratio from 20 at 20 weeks to 12 at 40 weeks

- signifying the relative predominant synthesis of OE at term.

- Discussion of Āccepted Conclusions -

- $\quad \mathrm{PR}$ is a metabolic intermediary product in OE synthesis. So whenever OE synthesising cell is stimulated, both hormones are produced. So

- Quantitatively OE and PR production predicts the number of OE synthesising cells of feto-placental unit i.e.

- Production increases with increase in the number of $\mathrm{OE}$ synthesing cells and vice-versa .

- Qualitatively maturation of OE synthesising cells is predicted by PR/OE ratio.

- i.e. lower PR/OE ratio shows OE predominance predicting better maturation of OE synthesising cells and viceversa.

- With the above well accepted observations, it can be concluded that foetal organogenesis and feto-placental steroidogenesis in a positive feedback mechanism help 
each other.

New Perspective Conclusion -

- $\mathrm{HCG}$ is inversely related to OE synthesis.

- Intensified OE and PR synthesis with PR/OE ratio of 12 at term predicts the maturation and viability of foetus.

- At term the negative feedback effect of dominant intensified OE synthesis on HCG inhibits feto-placental synthesis of $\mathrm{OE}$ and PR.

- The first dip is observed in PR level as the PR $t / 2$ is $30 \mathrm{~min}$ as compared to OEt/2 $8 \mathrm{hrs}$.

- This dip in PR is responsible for onset of parturition by reversing the myometrial excitability with spark release of Calcium ions.

Other Supporting Evidences .

- Ii] Studies On Pre-eclampsia showed increased HCG with decreased $\mathrm{OE}$ and $\mathrm{PR}$ along with increased PR/OE ratio as compared to values of normal gestation. $\left.{ }^{[24-25}\right]$

- There is inverse relation of $\mathrm{HCG}$ to $\mathrm{OE}$ and $\mathrm{PR}$, with relative dominance of PR in pre-eclampsia. i.e. HCG is 2-3 times more with25 - 50\% less OE and PR synthesis with high $\mathrm{PR} / \mathrm{OE}$ ratio.

- These fluctuations proportionately vary with the severity of pre-eclampsia.

New Perspective Conclusion In Pre-Eclamsia-

- Observations in pre-eclampsia proves the fact that

- there is decrease in the total number and

- maturation of OE synthesising cells of Feto-placental unit.

- This explains the increased association of IUGR [Intra uterine Growth Retardation] in pre-eclamsia i.e. reduced fetal organogenesis and feto-placental steroidogenesis. [24-25]

The Summary Of The New Perspective Conclusions In Answering The Following Questions.

- I] How body recognises the viability of fetus ?

- Quantitative increase in OE i.e.20 times more as compared to $4^{\text {th }}$ week value.

- Qualitative OE predominance. i.e. PR/OE ratio of 12 at term compared to 25 at $4^{\text {th }}$ week.

\section{CONLUSION -}

It indicates adequate $\mathrm{OE}$ synthesis predicting full fetal maturity.

- II] How feto-placental unit responds to full viable fetal status at term?

- Adequate OE by negative feedback causes inhibition of $\mathrm{HCG}$ preventing further $\mathrm{OE}$ and $\mathrm{PR}$ synthesis.

- III] Inspite of inhibition of both OE and PR why PR dip is first observed with peak of OE at term i.e. 24 - $48 \mathrm{hrs}$ before the normal parturition?

- Biological half-life of PR is very short i.e. 3 -30 minutes compared to 6-8 hrs of $\mathrm{t} / 2$ for $\mathrm{OE}$.

\section{The Observational Proofs Of PR Dip I.e.PR Withdrawal}

- a.Prominent PR dip is observed in animals ${ }^{[26]}$ and

- b.Increased urinary excretion of PR metabolites observed in human ${ }^{[26]}$

- c.Fall in BBT $1^{\circ} \mathrm{C}$ in calfs and bitch $24 \mathrm{hrs}$ before noticed by Concanon et al in $1989^{[17]}$

- d.In mares - low OE and PR observed near term but 5a PR metabolites production increased near term as observed by Holtan et al in 1975 and 1991. ${ }^{[18-19]}$

- e.OUSEY et al in 1884 observed high $\mathrm{Ca}$ and $\mathrm{Na}$ in prepartum milk before parturition which was used as indicator of parturition in a commercially used predictor kit. ${ }^{[20]}$

New Perspective Conlclusion - [Figure No. 3]

- At full term, due to negative feedback effect of OE on HCG there is diminished synthesis of OE and PR. As the half-life of circulatory PR is 3-30 min compared to $6-8 \mathrm{hrs}$ of $\mathrm{OE}$, the dip in PR level is first observed. The sudden PR withdrawal inducing acute respiratory acidosis increase biologically active plasma calcium ions which removes the 'progesterone block' of pregnancy, initiates parturition by facilitating myometrial contractility".

4] PR fluctuations at term reverse 'progesterone block' of pregnancy and initiate parturition with plasma calcium level oscillations facilitating myometrial contractility [figure no 3]

Mechanism of initiation and maintenance of parturition i.e. $1^{\text {st }}$ stage of labour pains due to maternal PR fluctuations with resultant active $\mathrm{Ca}^{2+}$ ions oscillations -

- At term, placental PR synthesis is inhibited $\rightarrow \downarrow P R[t / 2$ $=30 \mathrm{~min}] \rightarrow$ Acute respiratory acidosis $\rightarrow \uparrow$ release of biologically active plasma $\mathrm{Ca}^{2+}$ ions from inactive, plasma protein bound, storage forms $\rightarrow \uparrow \uparrow$ myometrial excitability $\rightarrow$ myometrial contraction and placental blood vessels contraction

- Placental vascular contraction and sandwitching of placenta between fetal membranes and uterus $\rightarrow$ squeeze out PR from placental store into maternal circulation

- $\uparrow \mathrm{PR}$ [t/2 =30min] $\rightarrow$ Acute respiratory alkalosis $\rightarrow \uparrow$ conversion of biologically active plasma $\mathrm{Ca}^{2+}$ ions to inactive store form bound to plasma proteins $\rightarrow \downarrow \downarrow$ myometrial excitability $\rightarrow$ myometrial relaxation and placental vascular relaxation.

- As placental PR synthesis is already inhibited, it again leads to $\downarrow$ in circulatory $\mathrm{PR}$ [t $/ 2=30 \mathrm{~min}] \rightarrow$ Acute respiratory acidosis $\rightarrow \uparrow$ release of biologically active plasma $\mathrm{Ca}^{2+}$ ions from calcium store bound to plasma proteins. $\rightarrow \uparrow \uparrow$ myometrial excitability $\rightarrow$ myometrial contraction

- Cycle of alternate uterine contraction and relaxation continues till placental PR store gradually exhausts $\rightarrow$ with gradual progress of myocardial excitability and ischaemic inflammatory response.

5] PR withdrawal induced facilitation of myometrial and cervical excitability establishes Oxytocin mediated neuroendocrinal reflex.

- Animal expt and clinical evidences of initiation of labour by Oxytocin ${ }^{[26]}$

6] Highlights of physiological importance of PR cyclical fluctuations along with biologically active Ca2+ oscillations during normal partutition

- Gradual progress in strength and duration assures prevention of fetal asphyxia by intermittent relaxation of placental blood vessels.

- It helps in minimizing maternal and fetal mortality and morbidity possible due to sudden PR withdrawal.

Maternal Morbidity And Mortality Will Be Increased In The Absence Of Controlled Regulation Of Active Ca2+ Ions Release. It Is Due To Spark Release Of Biologically Active Plasma $\mathrm{Ca}^{2+}$ On Sudden Pr Withdrawal [e.g. On Placental Separation As In Precipitus Delivery/ Caesarian Section] [fig No. 5]

- 1] Cardiac arrest due to Calcium rigor state.

- 2] Thrombo-embolism leading to morbidity and mortality

- 3] Even a slight PPH along with vaso-constriction of Hypothalamo-hypophyseal portal circulation leads to ischaemic necrosis resulting in Pan-hypo-pituitarism.

- 4] Generalized vaso-constriction causes sharp fall in BBT [Basal Body Temperature] leading to shivering.

- 5] Generalized convulsions due to suddenly enhanced CNS excitability

- 6] Puerperal psychosis.

- Foetal Morbidity And Mortality Will Be Increased Due 
To Insufficient Active Calcium Ions In Fetus [e.g. Caesarian Section/ Preipitus Delivery Due To Multiparity/Incompetent Cervix / Prematurity ]

- 1] Generalized myo - neural depression along with cardiorespiratory depression leads to asphyxia and related mortality and morbidity.

- 2] Low APGAR score at birth i.e. related to Appearance, Pulse, Grimace, Activity and Respiration.

- 7] Possible Predictions Of Parturition At Term.

- A] Breath Holding Time [bht In Seconds] At Tlc [total Lung Capacity] -

- Decreased BHT with normal Respiratory rate [RR] is indicator for sensitized Respiratory center by PR during pregnancy.

- Sudden increase in BHT with normal RR is predictor for PR withdrawal.

- B] BBT -

- There is $\mathrm{l}^{\circ} \mathrm{C}$ rise in $\mathrm{BBT}$ during pregnancy due to $\mathrm{PR}$ induced generalized vasodilatation.

- Sudden fall in BBT is predictor for PR withdrawal.

- C] Changes In pH Of Body Fluids With Associated Ca2+ Ions E.g. In Plasma, Urine, Saliva And Milk.

- There is acute and chronic respiratory alkalosis during pregnancy due to $P R$ induced respiratory center stimulation.

- Sudden change of urine and plasma $\mathrm{pH}$ from alkaline to acidic is predictor for $\mathrm{PR}$ withdrawal.

- Blood gas analysis for $\mathrm{pH}$ variation shows sudden change from respiratory alkalosis of pregnancy to acute respiratory acidosis with increased $\mathrm{pCO} 2$ and relative increase in calcium ions.

- 8] Further possible studies to strengthen the new perspective

- The above predictors can be studied with continuous monitoring of cardio-respiratory changes during normal parturition, pre-eclamsia etc.

\section{CONCLUSION -}

Awareness of importance of gradual intermittent release of placental PR and spark plasma $\mathrm{Ca}^{2+}$ helps clinicians in prevention of feto-maternal mortality and morbidity including puerperal psychosis associated with clinical conditions of sudden PR withdrawal as in caesarean section, precipitous labour, premature labour, preeclamsia etc. which can be treated by building better body blood buffers.
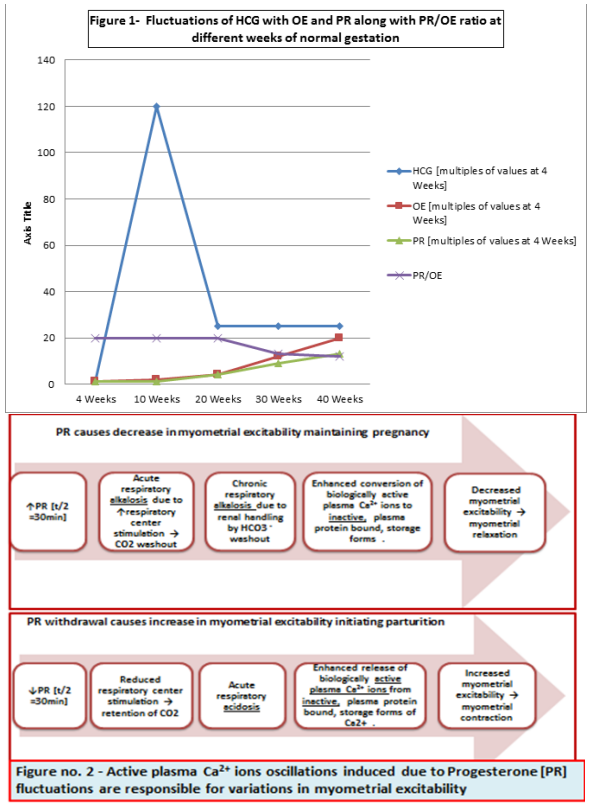
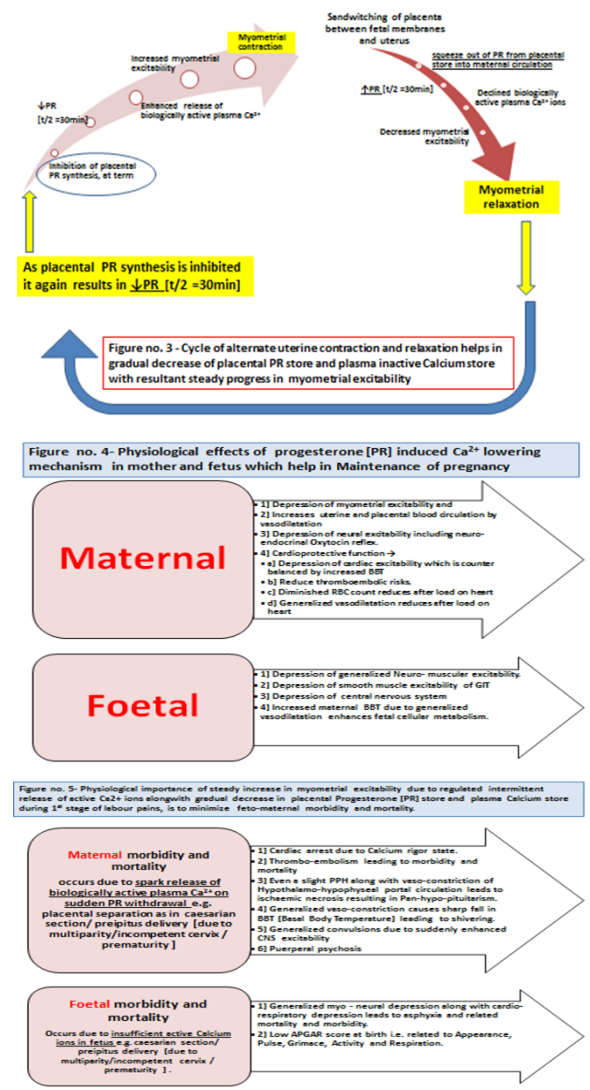

\section{REFERENCES -}

1. Volume 2 Sam Mesiano, ... Louis J. Muglia, in Knobil and Neill's Physiology of Reproduction (Fourth Edition), 2015

2. Handb Clin Neurol. 2014;124:17-36. doi: 10.1016/B978-0-444-59602-4.00002 2. Neuroendocrinology of pregnancy and parturition. Voltolini Cl, Petraglia F2.

3. Endocr Rev. 2010 Dec;31(6):783-816. doi: 10.1210/er.2009-0019. Epub 2010 Jul 14. Neuroendocrine mechanisms in pregnancy and parturition. Petraglia Fl Imperatore $A$, Challis JR.

4. PLoS One. 2015 Nov 24;10(11):e0143243. doi: 10.1371/journal.pone.0143243. eCollection 2015. High-Throughput Screening of Myometrial CalciumMobilization to Identify Modulators of Uterine Contractility. Herington JL1, Swale DR2, Brown N1, Shelton EL1, Choi H3, Williams CH4, Hong CC5,6, Paria BCl, Denton JS6,7, Reese Jl, 4

5. Front Biosci. 2007 Jan 1;12:619-33. Oxytocin and parturition: a role for increased myometrial calcium and calcium sensitization? Arthur Pl, Taggart MJ, Mitchell BF.

6. Physiology of parturition Author: Errol R Norwitz, MD, PhD, MBA, Section Editor: Charles J Lockwood, MD, MHCM; Deputy Editor: Vanessa A Barss, MD, FACOG

7. Pregnancy and Parturition; Jane E. Mijovic, David M. Olson, in Advances in Organ Biology, 1996

8. Reproductive and Endocrine Toxicology; A.R. LaBarbera, in Comprehensive Toxicology, 2010

9. The Endocrinology of Human Pregnancy and Fetoplacental Neuroendocrine Development; Sam Mesiano, in Yen \& Jaffe's Reproductive Endocrinology (Sixth Edition), 2009

10. Reproductive anatomy and physiology; Timothy J. Evans, Vekataseshu K Ganjam, in Reproductive and Developmental Toxicology, 2011

11. Rabbit Colony Management and Related Health Concerns ; Robert H. Quinn, in The Laboratory Rabbit, Guinea Pig, Hamster, and Other Rodents, 2012

12. Dharwadkar Anand R et al. "Negative Feed Back Regulation of Oestrogen \& Vasodilatory Function of Progesterone Responsible for Preovulatory Gonadotropin Surge [LH Surge] - A Hypothesis". JMSCR Volume 3 Issue 4 Page 5089-5094: April 2015. 30.

13. Dharwadkar AA, Chenmarathy BB, Dharwadkar AR. "A Comparative Study of breath holding time as an Index of Central Ventilatory Response in young Healthy Adults of both Sexes". J Pharm Biomed Sci 2014;04(09):806-812.

14. Dharwadkar Asha A., Dharwadkar Anand R., Chenmarathy Bindu B. Impact of three periovulatory progesterone fluctuations in ovulation and tubal suction of released ovum: A new perspective. International journal of scientific research. Volume-6 | Issue-7 | July-2017 | ISSN No 2277 - 8179 | IF : 4.176 | IC Value : 78.46 available https://www.worldwidejournals.com/internationaljournal-of-scientific-research-(IJSR)//articles.php?val =MTEOMD $=\& \mathrm{bl}=777 \& \mathrm{k}=195$

15. Dharwadkar AA, Chitterusu RR, Dharwadkar AR, Chenmarathy BB, Dharwadkar KA. Cardioprotective function of progesterone: A new perspective. Natl J Physiol Pharm Pharmacol. (2017), [cited July 20, 2017]; 7(2): 136-142. doi:10.5455/njppp.2017.7.0927404102016available https://www.n njppp.com/?jid=28\&iid =2017-7-2.000

16. Dharwadkar Asha A., Dharwadkar Anand R., Chenmarathy Bindu B. intra spermic calcium store in reversible state of calcium rigor is responsible for 
sperm motility and polyspermy prevention -A new prespective. International Journal Of Basic And Applied Physiology, 2017; 6[1]: 1-10

17. Concannon PW (2002). Physiology and clinical parameters of pregnancy in dogs. 27 Proc WorldSmall Anim Vet Assoc Granada Spainhttp://www. vin.com /proceedings

18. Holtan DW, Nett TM, Estergreen VL (1975).Plasma progestagens in pregnant mares. J Reprod Fertil Suppl 23: 419-24.

19. .Holtan DW, Houghton E, Silver M et al. (1991).Plasma progestagens in the mare, fetus and newbornfoal. J Reprod Fertil Suppl 44: 517-28.

20. Ousey JC, Dudan FE, Rossdale PD (1984a).Preliminary studies of mammary secretions in themare to assess fetal readiness for birth. Equine Vet $J 16: 259$ 63.

21. Barret KE, Barman SM, Boitano S, Brooks H. Ganong Review of Medical Physiology. 24th ed. Ch. 22- 23. New Delhi: Tata McGraw-Hill Education Private Limited Publication; 2012. Chapter 22-23, 391 -419.

22. Guyton -ref Hall JE: Guyton \& Hall Text book of Medical Physiology. 12th ed.Ch 96, 98. South Asian Edition: Elsevier Inc. Philadelphia; 2011. 505-507, 636 -650, 656.1030-1038, [Figure 96-9].

23. Guyton AC, Hall JE. Text Book of Medical Physiology. 1lth ed. Ch. 82. Philadelphia, PA: Elsevier Inc.; 2006. P. 68, 92-100, 514-520, 978- 980,10111025, 383-398, 505, 507, 514-525,1030-1038, [Fig 82-7] page no.1032

24. Suaad A. Brakhas, Abbass M. Rahmah, Amna N.Jassim "Evaluation the Levels of Serum Hormones (Progesterone, Estradiol, and hCG) in Preeclamptic Iraqi Pregnancies" Baghdad Science Journal Vol.9(2)2012

25. BEGUM Z1, ARA I2, TANIRA S3, KEYA KA4. - "The association between serum betahuman chorionic gonadotropin and preeclampsia": J Dhaka Med Coll. 2014; 23(1): 89-93.

26. Purohit G. Parturition In Domestic Animals: A Review. WebmedCentral REPRODUCTION 2010;1(10):WMC00748T 